Bull. Mater. Sci., Vol. 22, No. 3, May 1999, pp. 717-721. (C) Indian Academy of Sciences.

\title{
Micro-parameters and micro-characteristics related to the formation of $\varepsilon$-martensite in Fe-based shape memory alloys
}

\author{
BOHONG JIANG* and XUAN QI \\ Open Lab of State Education Commission for High-Temperature Materials and Testing, Shanghai Jiao Tong \\ University, Shanghai 200030, China
}

\begin{abstract}
The influence of some microstructure parameters and characteristics of $\boldsymbol{\gamma}$-phase e.g. Stacking fault probability, grain size and texture etc on $\varepsilon$-martensitic transformation, shape memory effect and other macro-behaviours in $\mathrm{Fe}-\mathrm{Mn}-\mathrm{Si}$ based alloys are reviewed.
\end{abstract}

Keywords. Fe-Mn-Si; shape memory effect; $\varepsilon$-martensitic transformation; stacking fault probability; grain size; texture.

\section{Introduction}

Recently Fe-Mn-Si based alloys, a newly developed shape memory alloy system, are attracting great attention both in theory and practice owing to their good shape memory effect (SME) and cheap cost as well as fine workability (Sato et al 1982; Otsuka et al 1990). As in TiNi, Cu-based and other SMAs, we can find some microstructure parameters, which closely relate to the martensitic transformation, SME and other macrobehaviour of alloys. By virtue of the particularity of the phase transformation there are distinguishing micro parameters peculiar to the different alloy systems. For example, in $\mathrm{Cu}-\mathrm{Zn}-\mathrm{Al}$ alloys the ordering of parent phase is one of the necessary conditions for obtaining a good SME. We have deduced that the $M_{\mathrm{s}}$ temperature is a function of ordering parameter $\xi^{2}$ in $\mathrm{Cu}-\mathrm{Zn}-\mathrm{Al}$ alloys (Wu et al 1988) and the SME is closely related with the ordering degree (Jiang and Hsu 1990). However, it is not the case in Fe-based alloys because there is no ordering transformation in these alloys. In this paper several of our recent works dealing with the effect of micro-parameters and characteristics on the macrobehaviours related to the $\varepsilon$-martensitic transformation and $\mathrm{SME}$ in $\mathrm{Fe}-\mathrm{Mn}-\mathrm{Si}$ based alloys are summarily reviewed.

\section{Stacking faults}

It is well known that $\mathrm{Fe}-\mathrm{Mn}-\mathrm{Si}$ based alloys undergo a phase transformation from $\gamma$ (fcc) to $\varepsilon$ (hcp) structure, and the $\varepsilon$-phase can be formed by means of stacking faults which are introduced on every second layer of the $\{111\}$ planes in austenite. The $\varepsilon$-martensite can be formed thermally-induced and stress-induced. In both

*Author for correspondence cases the stacking faults will play an important role. For example, the nucleation of $\varepsilon$-phase can be realized by the multiplication of stacking faults through the sweeping of the partial dislocation around a pole dislocation (Seeger 1956; Hoshino et al 1990) or directly by the overlapping of stacking faults (Olson and Cohen 1976; Hsu 1980), which will affect the characteristics of the phase transformation, e.g. $M_{\mathrm{s}}$ temperature, the critical stress required for inducing $\varepsilon$-martensite, $\sigma_{\mathrm{M}}$, as well as mechanical behaviour and SME.

The characteristics of stacking faults in $\mathrm{Fe}-\mathrm{Mn}-\mathrm{Si}$ based alloys are extremely wide usually extending from one side of a parent grain boundary to the other side as observed in TEM, because of relative low level of the stacking fault energy in these alloys. The extending dislocations with limited width and the extending nodes of stacking faults which are easily observed in stainless steels, Ni-based and other alloys can not be found in $\mathrm{Fe}-\mathrm{Mn}-\mathrm{Si}$ based alloys. Therefore, it is difficult to evaluate the stacking fault energy by measuring the width of extending dislocations or the extending node radii from TEM.

By means of an $\mathrm{X}$-ray diffraction profile analysis the stacking fault probability can be determined and then the stacking fault energies can be estimated for several metals (Reed and Schramm 1974).

\subsection{Stacking fault probability}

Fourier analysis of X-ray diffraction profile for polycrystal with stacking fault indicates that there are three diffraction effects of stacking fault: the broadening and asymmetry of profile as well as the displacement of diffraction peak (Warren 1969). In our works, the stacking fault probability, $P_{\mathrm{sf}}$, was determined by the broadening effect of diffraction profile (Qi et al 1998). 
The stacking fault energy SFE can be estimated by following approximate equation (Noskova and Pavlov 1962):

$$
\mathrm{SFE}=\frac{G \cdot a_{0}^{2} \cdot d \cdot \rho}{24 \pi \cdot P_{\mathrm{sf}}},
$$

where $G$ is the shear modulus, $a_{0}$ the lattice parameter, $d$ the lattice space of determined crystal plane and $\rho$ the dislocation density. The error of above (1) is relatively large, because the calculation of $\rho$ needs several hypothesis models. Another equation for estimating SFE from stacking fault probability (Reed and Schramm 1974) is also an approximate one because of the arbitrary nature of the selection in the proportional constants.

Therefore, the SFE can only be estimated approximately by above equations. Actually in most cases we need only the relative variation rule of SFE in matrix with various external conditions such as alloying elements, heat-treatments etc. Accordingly in present work we only measure the $P_{\mathrm{sf}}$ for relative comparison, since the $P_{\mathrm{sf}}$ is inversely proportional to $\mathrm{SFE}$, i.e. SFE $\propto 1 / P_{\mathrm{sf}}$.

\subsection{Effect of alloying composition on $P_{s f}$}

The stacking fault probability, $P_{\text {sf }}$ of $\mathrm{Fe}-\mathrm{Mn}-\mathrm{Si}$ specimens with various alloying compositions were determined and the starting temperature of $\gamma \rightarrow \varepsilon$ transformation, $M_{s}$, of alloys are measured. The results are listed in table 1 . It is shown that the addition of $\mathrm{Cr}$ and $\mathrm{N}$ reduces $P_{\text {sf }}$ and correspondingly decreases $M_{\mathrm{s}}$ as comparing alloy 0 with $\mathrm{M} 3$ as well as $\mathrm{N} 1$, respectively. While comparing alloy $\mathrm{M} 1$ to $\mathrm{M} 4$, the $P_{\mathrm{sf}}$ and $M_{\mathrm{s}}$ run lower with increasing content of $\mathrm{Mn}$.

Recently we have measured the stacking fault probability in stainless steel type SMAs i.e. $\mathrm{Fe}-14 \mathrm{Mn}-6 \mathrm{Si}-$ $13 \mathrm{Cr}-5 \mathrm{Ni}$ with different contents of cobalt and found that the $P_{\text {sf }}$ increases with increasing Co. The results agree with that reported in literature as the stacking fault energy measurement.

\subsection{Relationship between $M_{s}$ and $P_{s f}$}

We sum up the results of $M_{\mathrm{s}}$ points and $P_{\mathrm{sf}}$ from different heat treatments in various alloys listed in table 1 (Jiang et al 1998). While plotting $M_{\mathrm{s}}$ vs $1 / P_{\mathrm{sf}}$, a linear line is obtained by linear regression as shown in figure 1 :

$$
M_{\mathrm{s}}(K)=372-0 \cdot 113 / P_{\mathrm{sf}} .
$$

The above relationship can be deduced from thermodynamics. The change in the total free energy per unit volume associated with the $\gamma$ (fcc) $\rightarrow \varepsilon$ (hcp) martensitic transformation $\Delta G^{\gamma \rightarrow \varepsilon}$, may be expressed as

$$
\Delta G^{\gamma \rightarrow \varepsilon}=\Delta G_{c}^{\gamma \rightarrow \varepsilon}+\Delta U_{\mathrm{str}}+s / v(a+\xi),
$$

where $\Delta G_{c}^{\gamma \rightarrow \varepsilon}$ and $\Delta U_{\text {str }}$ refer to the changes of chemical free energy and coherency strain energy per unit volume, respectively, $a$ is the specific surface energy of hcp phase, $\xi$ the SFE of austenite, $s$ and $v$ are area and volume of the hcp phase formed, respectively. A calculation of the coherency strain energy change for $f c c \rightarrow h c p$ transformation in $\mathrm{Fe}-\mathrm{Cr}-\mathrm{Ni}$ alloy (Olson and Cohen 1976) revealed that this energy is $<0.1 \mathrm{pct}$ of the measured fault energy. Hence, the contribution of $\Delta U_{\text {str }}$ can be neglected here.

The $M_{\mathrm{s}}$ temperature can be defined as the temperature, $T$ at which $\Delta G^{\gamma \rightarrow \varepsilon}=0$. Thus, at $M_{\mathrm{s}}$ (3) becomes

$$
-\Delta G_{\mathrm{c}}^{\gamma \rightarrow \varepsilon}=s / v(a+\xi)=C+D / P_{\mathrm{sf}},
$$

where $C$ and $D$ are constants.

Owing to that $M_{\mathrm{s}}$ temperature varies within a relatively small range, the changes in enthalpy $\Delta H^{\gamma \rightarrow \varepsilon}$ and $\Delta S^{\gamma \rightarrow \varepsilon}$ can be taken as constants independent of temperature. Thus the chemical free energy difference accompanying $\gamma \rightarrow \varepsilon$ can be expressed as

$$
\Delta G_{\mathrm{c}}^{\gamma \rightarrow \epsilon}=\Delta H^{\gamma \rightarrow \varepsilon}\left(1-T / T_{0}\right),
$$

where $T_{0}$ is the temperature at which $\Delta G_{c}^{\gamma \rightarrow \ell}=0$.

When $T=M_{s}$, we obtain

$$
M_{\mathrm{s}}=T_{0}+\frac{\left(C+D / P_{\mathrm{sf}}\right) T_{0}}{\Delta H^{\gamma \rightarrow \varepsilon}}
$$

Owing to that $\Delta H^{\gamma \rightarrow \varepsilon}<0$, and for a given alloy, $\Delta H^{\gamma \rightarrow E}$ and $T_{0}$ are constants, thus

Table 1. Composition of the tested alloys (wt $\%$ ) and measured $P_{\mathrm{sf}}, M_{\mathrm{s}}$.

\begin{tabular}{lccccccc}
\hline Alloy & $\mathrm{Mn}$ & $\mathrm{Si}$ & $\mathrm{Cr}$ & $\mathrm{N}$ & $\mathrm{Fe}$ & $P_{\mathrm{sf}}\left(\times 10^{-3}\right)$ & $M_{\mathrm{s}}(\mathrm{K})$ \\
\hline 0 & 30.3 & 6.1 & - & - & balance & 1.83 & 309 \\
M1 & 26.4 & 6.2 & 5.2 & - & balance & 1.65 & 307 \\
M3 & 30.0 & 6.0 & 5.3 & - & balance & 1.17 & 258 \\
M4 & 32.2 & 6.4 & 5.2 & - & balance & 0.81 & 240 \\
N1 & 30.0 & 6.0 & - & 0.05 & balance & 1.60 & 304 \\
\hline
\end{tabular}

(Austenized at $1073 \mathrm{~K}$ for $0.5 \mathrm{~h}$ followed by water quenching) 


$$
M_{\mathrm{s}}=A-B / P_{\mathrm{sf}},
$$

where $A$ and $B$ are constants related to materials.

It is indicated from (7) that factors increasing $P_{\mathrm{sf}}$, will increase $M_{\mathrm{s}}$ and vice versa. Therefore in the present experiments, increasing the content of $\mathrm{Mn}$ or adding $\mathrm{Cr}$ or $\mathrm{N}$ in $\mathrm{Fe}-\mathrm{Mn}-\mathrm{Si}$ alloys will decrease $M_{\mathrm{s}}$ temperature.

\subsection{Relationship between $\sigma_{M}$ and $P_{s f}$}

The proof yield stress $\sigma_{0 \cdot 05}$ on the stress-strain curve obtained in extension test is taken as the critical stress required for inducing $\varepsilon$-martensite, $\sigma_{\mathrm{M}}$. Owing to that the $\sigma_{\mathrm{M}}$ is usually lower than the real yield stress at room temperature in these tested alloys.

While summing up all the $P_{\mathrm{sf}}$ and $\sigma_{\mathrm{M}}$ resulting from various tested alloys and different treat conditions, a linear relationship between $\sigma_{\mathrm{M}}$ and $1 / P_{\mathrm{sf}}$ is obtained by linear regression as shown in figure 2:

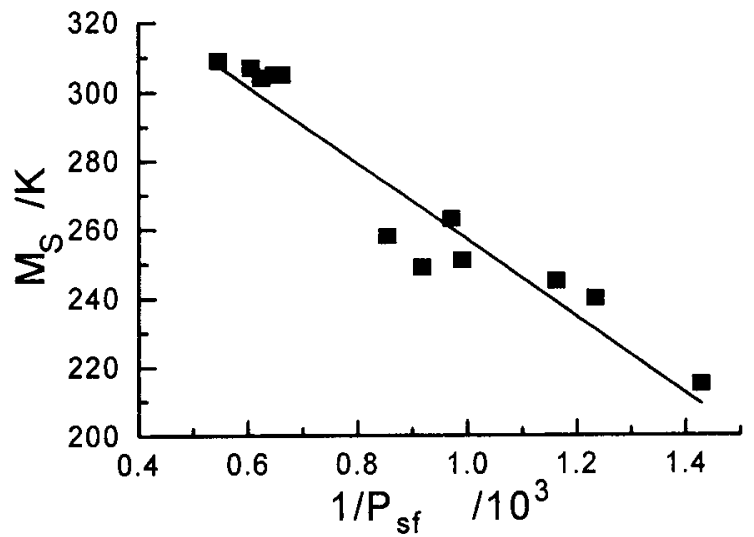

Figure 1. Variation of $M_{\mathrm{s}}$ temperature vs reciprocal of stacking fault probability $\left(1 / P_{\mathrm{sf}}\right)$ in $\mathrm{Fe}-\mathrm{Mn}-\mathrm{Si}$ based alloys.

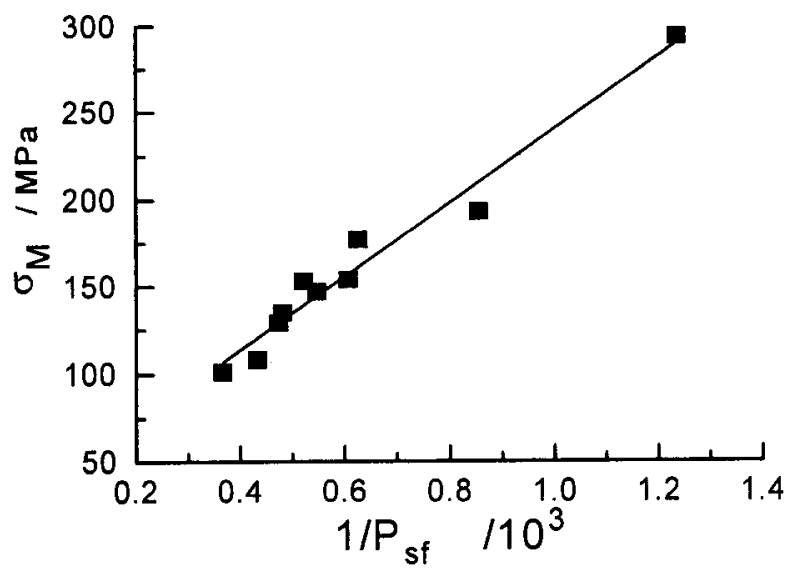

Figure 2. A linear relationship between $\sigma_{\mathrm{M}}$ and $1 / P_{\mathrm{sf}}$ in $\mathrm{Fe}-\mathrm{Mn}$-Si-based alloys by linear regression as $\sigma_{\mathrm{M}}(\mathrm{MPa})=$ $29+0.212 / P_{s f}$. The correlation coefficient is $\approx 0.98$.

$$
\sigma_{\mathrm{M}}(\mathrm{MPa})=29+0 \cdot 212 / P_{\mathrm{sf}} .
$$

The Gibbs free energy per mole volume of $\gamma \rightarrow \varepsilon$ transformation for stress-induction can be written as follows:

$$
\Delta G^{\gamma \rightarrow \varepsilon}=G_{\mathrm{c}}^{\gamma \rightarrow \varepsilon}+\Delta G_{\sigma}^{\gamma \rightarrow \varepsilon},
$$

where $\Delta G_{c}^{\gamma \rightarrow \varepsilon}$ is expressed as (13) and $\Delta G_{\sigma}^{\gamma \rightarrow \varepsilon}$ is the mechanical driving energy supplied by applied stress which can be written as (Tamura 1982)

$$
\Delta G_{\sigma}=\frac{1}{2} \sigma_{\mathrm{a}}\left[\varepsilon_{\tau} \sin 2 \theta \pm \varepsilon_{\mathrm{n}}(1+\cos 2 \theta)\right]
$$

where $\sigma_{\mathrm{a}}$ is the applied stress, $\varepsilon_{\tau}$ and $\varepsilon_{\mathrm{n}}$ are the shear strain and normal strain of phase transformation respectively; and $\theta$ is the orientation angle between stress-induced martensite and applied stress.

While the applied stress at deformation temperature, which is room temperature in the case of the present study, reaches a certain value, the total free energy $\Delta G^{\gamma \rightarrow \varepsilon}$ just equals to zero so that the motion of Schockley partial dislocations are activated and the stacking faults are extended and overlapped, thus a first plate of $\varepsilon$-martensite is induced, in which the applied stress is the critical stress required for inducing transformation, $\sigma_{\mathrm{M}}$. Thus, we obtain

$$
-\left[C+D / P_{s t}\right]+\frac{1}{2} \sigma_{M} \Phi=0 .
$$

Then

$$
\sigma_{\mathrm{M}}=M+N / P_{\mathrm{sf}},
$$

where $\Phi, M$ and $N$ are constants. Therefore the critical stress required for inducing martensite is proportional to the reciprocal of stacking fault probability, being in good agreement with the experimental results.

\subsection{Effect of thermally mechanical training on $P_{s f}$}

It has been pointed out by many investigators that thermal mechanical training can effectively improve the shape memory effect of the $\mathrm{Fe}-\mathrm{Mn}-\mathrm{Si}$ based alloys (Otsuka et al 1990; Inagaki 1992), but the mechanism is still not consistent. In our work the increase of stacking faults probability by training has been irrefutably proved by the X-ray profile analysis data as shown in table 2 . Correspondingly the data of shape recovery degree $\eta$ are also listed in table 2 . We suggest that the essential cause of improvement of SME by thermal mechanical training may be the increase of stacking faults, which will not only provide the nucleation sites for SIM on the next deformation that leads to the decrease of $\sigma_{\mathrm{M}}$ and the increase of the amount of stress-induced $\varepsilon$-martensite on the same strain level, but also lead to the increase of 
strain-hardening exponent that causes $\gamma$-phase strengthening homogeneously, resulting in the decrease of permanent strain; and on the other side promote the formation of finer lamella structure consisting of $\varepsilon$-martensite and residual austenite that will make the reverse transformation complete. All these facts will improve the shape recovery ability.

\section{Grain size of $\gamma$-phase}

Normally the grain size of $\gamma$-phase is an important microstructure parameter affected by phase transformations and mechanical properties. In $\mathrm{Ni}-\mathrm{Ti}$ and $\mathrm{Cu}$-based SMAs, the $M_{\mathrm{s}}$ temperature closely depends on the grain size. For example we have deduced that $M_{\mathrm{s}}$ is linearly related to the reciprocal of the square root of the grain size $d$ as,

$$
M_{\mathrm{s}}=M_{\mathrm{s}}^{\mathrm{s}}-K d^{-1 / 2},
$$

where $M_{\mathrm{s}}^{\mathrm{s}}$ is a constant instead of the $M_{\mathrm{s}}$ temperature of a single crystal of the concerned alloy, and $K$ a constant related to the materials (Wu et al 1988). However the above relation is not found in $\mathrm{Fe}-\mathrm{Mn}-\mathrm{Si}$ based SMAs. We have tested the $M_{s}$ of $\varepsilon$-martensitic transformation for treatment of different austenitizing temperatures, as shown in table 4 (Jiang et al 1996).

The results of table 3 indicate that the change in grain size of $\gamma$-phase has no marked influence on $M_{\mathrm{s}}$. The reason may be that in alloys with lower stacking fault energy, the nucleation of $\varepsilon$-martensite occurs by means of the pre-existed stacking faults rather than through nucleates at grain boundaries (Hsu 1980), which is quite different from the result. In Cu-based alloys, the item of elastic strain energy becomes a main resistance against the chemical driving force in the thermodynamic equi- librium equation. The shear stress required for the formation of $\alpha$-martensite increases with decreasing the

Table 2. Effect of thermal mechanical cycling of alloy N1.

\begin{tabular}{lccc}
\hline Cycle no & $\sigma_{\mathrm{M}}(\mathrm{Pa})$ & $\eta(\%)$ & $P_{\mathrm{sf}}\left(\times 10^{-3}\right)$ \\
\hline 1 & 177 & 43 & 1.60 \\
2 & 153 & 79 & 1.92 \\
3 & 135 & 90 & 2.08 \\
4 & 126 & 99 & - \\
5 & 108 & 100 & 2.31 \\
\hline
\end{tabular}

grain size which is related to the strength of the parent phase through the Hall-Petch relation (Wu et al 1988). However the item of elastic strain energy for fcc $\rightarrow$ hcp transformation is so small as to be neglected as mentioned above. The main resistance becomes SFE, which is independent of the grain size.

\section{Texture of $\gamma$-phase}

The origin of SME of Fe-Mn-Si alloys is the reversion of macro deformation during heating, which is produced by the preferred stress-induced martensite or the preferred orientation of martensitic variants under applied stress. Obviously the preferred alignment of martensites is a texture problem in phase transformation. The component extent of texture will directly relate to SME. For example, extending a single crystal of Fe-Mn-Si alloy along (414) direction a maximum recoverable strain of $20 \%$ can be obtained (Sato et al 1982). Normally, however, polycrystal materials industry used $\mathrm{FeMnSi}$, the recoverable preferential strain $\varepsilon_{\mathrm{R}}$ only reached $2 \%$. Evidently it is related to the preferential extent of the stress-induced martensite. Otsuka et al (1990) have presented a formula for evaluating $\varepsilon_{\mathrm{R}}$

$$
\varepsilon_{\mathrm{R}}=20 \cdot f\left(V_{\varepsilon} \%\right)
$$

where $f$ is a preferred factor and $V_{\varepsilon} \%$ is the amount of SIM.

Recently we have studied the textures of $\gamma$-phase in an annealed $\mathrm{Fe}-27 \mathrm{Mn}-6 \mathrm{Si}-5 \mathrm{Cr}$ alloy wire by ODF analysis and connect with shape memory degree (Qi et al 1998). It is found that both the component and extent of fibre texture are variable with different annealing temperatures. For example, there are four kinds of texture i.e. $\langle 001\rangle,\langle 111\rangle,\langle 011\rangle$ and $\langle 221\rangle$ for annealing at $873 \mathrm{~K}$. The intensity of these texture components is nearly the same except that of $\langle 221\rangle$ which is lower. However, after annealing at $973 \mathrm{~K}$ the $\langle 001\rangle$ texture becomes very strong, and the $\langle 011\rangle$ and $\langle 221\rangle$ components disappear while the $\langle 111\rangle$ one only remains a trace. For annealing at $1073 \mathrm{~K}$ the above four components appear together again, while the intensities are all lower. After annealing at $1123 \mathrm{~K}$ only $\langle 001\rangle$ and $\langle 011\rangle$ can be detected.

The shape recovery degree $\eta$, which is determined by extending the wire specimen followed by recovery heating to $873 \mathrm{~K}$, varies with annealing temperature as shown in table 4.

Table 3. $M_{\mathrm{s}}$ of $\gamma \rightarrow \varepsilon$ for different austenitizing temperature in Fe-26.4 $\mathrm{Mn}-6 \cdot 2 \mathrm{Si}-5 \cdot 2 \mathrm{Cr}$ (wt \%).

\begin{tabular}{lllllll}
\hline Austenitizing temperature (k) & 1073 & 1123 & 1173 & 1223 & 1273 \\
\hline Mean grain size of $\gamma(\mu \mathrm{m})$ & 20.62 & 23.70 & 28.30 & 34.58 & 37.12 \\
$M_{\mathrm{s}}$ temperature $(\mathrm{k})$ & 307 & 311 & 311 & 313 & 313 \\
\hline
\end{tabular}


Table 4. Shape recovery degree at various annealing temperatures.

\begin{tabular}{lllll}
\hline Temperature (K) & 873 & 973 & 1073 & 1123 \\
\hline$\eta(\%)$ & 47 & 65 & 40 & 38 \\
\hline
\end{tabular}

It is indicated that the best shape recovery degree is obtained by annealing at $973 \mathrm{~K}$ corresponding to the heat treatment of getting nearly monomial texture. Obviously there is some connection between SIM and texture. The calculation of the angles between the fibre texture direction $\langle u v w\rangle$ and the moving direction of $1 / 6\langle 211\rangle$ partial dislocations shows that extending along $\langle 001\rangle$ direction the maximum shear stress direction will be the nearest one to the stress induced direction. Therefore the more the crystal grains align with $\langle 001\rangle$, the easier the $\varepsilon$-martensites form.

\section{Conclusions}

There are some micro-parameters and micro-characteristics which affect the macro-behaviours such as the phase transformation temperature of $\varepsilon$-martensite and SME in Fe-Mn-Si based shape memory alloys and can be summarized as follows:

(I) Stacking fault probability $P_{\mathrm{sf}}$ as a micro-parameter takes an important effect on both $M_{\mathrm{s}}$ temperature and $\sigma_{\mathrm{M}}$, the critical stress of SIM. The larger the $P_{\mathrm{sf}}$, the higher the $M_{\mathrm{s}}$ and the lower the $\sigma_{\mathrm{M}}$. The thermal mechanic cycling will increase $P_{\mathrm{sf}}$ so that it decreases $\sigma_{\mathrm{M}}$.
(II) The grain size of parent phase has no obvious influence on the $M_{\mathrm{s}}$ temperature for the fcc to hcp transformation.

(III) The texture of parent phase acts as a non-negligible role on the shape memory effect.

\section{References}

Hoshino Y, Nakamura S, Ishikawa N and Sato A 1990 Mater. Sci. Forum 56-58 643

Hsu T Y (Xu Zuyao) 1980 Acta Metall. Sinica 16430 Inagaki H 1992 Z. Metallkd 838097

Jiang B H and Hsu T Y (Xu Z Y) 1990 Mater. Sci. Forum 56-58 457

Jiang B H, Qi X, Zhou W M and Hsu T Y (Xu Z Y) 1996 Scr. Metall. et Mater. 34771

Jiang $B \mathrm{H}$, Qi $X$, Yang $\mathrm{S} X$, Zhou $\mathrm{W} M$ and $\mathrm{Hsu} T \mathrm{~T}$ (Xu Z Y) 1998 Acta Mater. 46501

Noskova N I and Pavlov V A 1962 Phys. Metals \& Metall. 14899

Otsuka H et al 1990 Iron Steel Inst. Japan Int. 30674

Olson G B and Cohen M 1976 Metall. Trans. A7 1897

Qi X, Jiang B H and Hsu T Y (Xu Zuyao) 1998 Physical Testing and Chemical Analysis, Part A: Physica Testing, 34 16 (in Chinese)

Reed R P and Schramm R E 1974 J. Appl. Phys. 454705

Sato A, Chishima E, Soma K and Mori T 1982 Acta Metall. 301177

Seeger A 1956 Metallkunde 47653

Tamura I 1982 Metal Sci. 16245

Warren B E 1969 X-ray diffraction (Reeding, Mass: AddisonWesly) pp 275-294

Wu J X, Jiang B H and Hsu T Y (Xu Z Y) 1988 Acta Metall. 361521 